\title{
MODEL STUDIES OF THE VIBRATIONS OF STRUCTURES DURING EARTHQUAKES, BASED ON GROUND ACCELERATIONS INSTEAD OF ON GROUND DISPLACEMENTS*
}

\author{
By Merit P. White and Ralph Byrne
}

ONE of the most important problems in the field of earthquake engineering deals with the dynamics of structures. Any building is an elastic system. As such, its deflections (stresses) during an earthquake may depend on its own dynamical characteristics as well as on the nature of the ground motion. An ordinary building is a relatively complicated dynamic system, usually of many degrees of freedom and with considerable internal friction. The treatment of this problem may be simplified by the assumption that this internal friction is equivalent to viscous damping. ${ }^{1}$ A further simplification results from first treating systems of one degree of freedom, combining these results if the behavior of a more complicated system is desired.

Although very useful conclusions can be drawn with no knowledge of actual ground motions, nevertheless the most valuable results will be based on records of actual earthquakes, such as the accelerograms recently obtained in California and Montana by the United States Coast and Geodetic Survey.

The response of a given oscillator to a particular disturbance may be obtained analytically or experimentally. The usual, in fact to date the only, experimental procedure has been to subject the oscillator model to a base displacement corresponding to the one being studied. Analytical calculations of the response, on the other hand, can be based on ground accelerations or velocities as well as displacements. This fact has given a certain advantage to the analytical approach, since the principal records of severe ground motions are acceleration records which must be double-integrated if displacements are desired.

However, it is possible to carry out model studies of linear, one degree of freedom oscillators with or without viscous damping, using only ground accelerations. This paper describes the general procedure which must be followed and gives the argument justifying this process.

Consider first a simple, undamped, linear oscillator consisting of a mass on a flexible support whose base is being accelerated at the rate $j$. We introduce the following expressions used in the equations below:

$$
\begin{aligned}
j & =\text { ground acceleration } \\
m & =\text { moving mass } \\
y & =\text { deflection of spring }
\end{aligned}
$$

* Manuscript received for publication September 14, 1938.

${ }^{1}$ For justification of this assumption, see page 124 (Chap. VII, "The Building and Ground Vibrator," by J. A. Blume) of U. S. Dept. Commerce, Special Publ. no. 201, "Earthquake Investigations in California, 1934-1935" (1936). 


$$
\begin{aligned}
\ddot{y} & =\frac{d^{2} y}{d t^{2}} \\
k & =\text { spring constant (force to produce unit deflection) } \\
\lambda^{2} & =k / m \\
T_{0} & =2 \pi / \lambda \text { (natural vibration period of undamped system) }
\end{aligned}
$$

From equilibrium of forces we obtain the equation of motion:

$$
\ddot{y}+\lambda^{2} y=-j
$$

If $j$ is not a rational function of time, equation (1) can be solved in the following way. Imagine that the system is at rest up to time $t=0$, at which instant

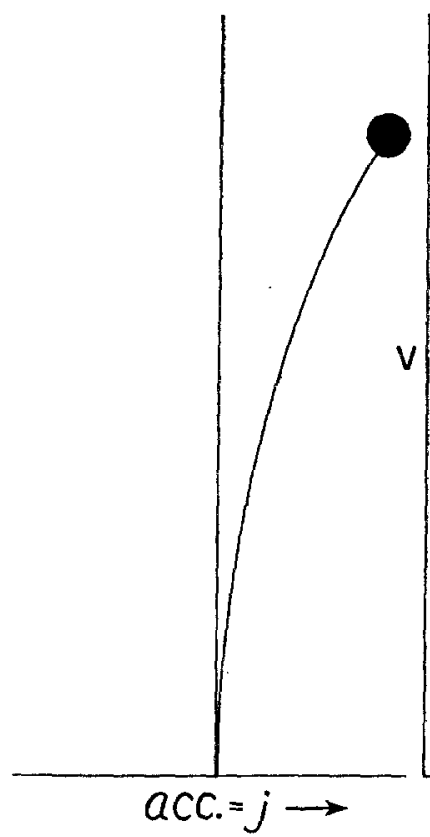

Fig. 1

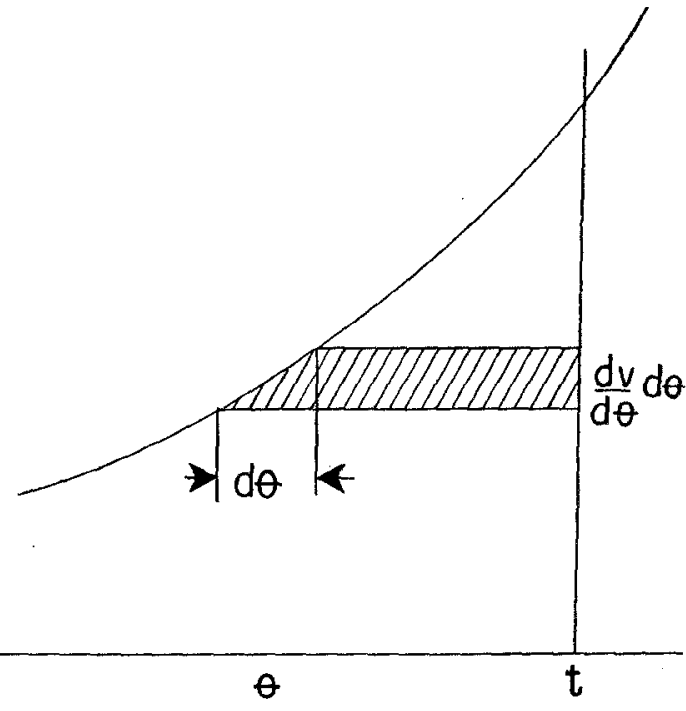

Fig. 2

a constant velocity $v_{0}$ is applied to the base $(j=0$, except at the instant $t=0)$. We can find the motion (i.e., the response to an increment of velocity) by solving equation (1) (with $j=0$ ) subject to the boundary conditions:

$$
\text { at } t=0 \quad \begin{aligned}
(y)_{0} & =0 \\
(\dot{y})_{0}+v_{0} & =0
\end{aligned}
$$

That is, $m$ is at rest in space at the initial instant. The solution of equation (1) for the boundary conditions given above is:

$$
y=-\frac{v_{0}}{\lambda} \sin \lambda t
$$


Consider now the velocity diagram shown in figure 2. From equation (2) it follows that at time $t$ the effect on $y$ of an increment of velocity $\frac{d v}{d \theta} d \theta$ acting at time $\theta$ (its effect indicated by the shaded strip in figure 2 ) will be:

$$
\begin{aligned}
d y & =-\frac{1}{\lambda} \frac{d v}{d \theta} d \theta \sin \lambda(t-\theta) \\
& =-\frac{j}{\lambda} \sin \lambda(t-\theta) d \theta
\end{aligned}
$$

At any time $t$, the cumulative effect of all previous velocity increments is found by integrating the equation just given with respect to $\theta$ between the limits 0 and $t$. This gives:

$$
y(t)=-\frac{1}{\lambda} \int_{0}^{t} j(\theta) \sin \lambda(t-\theta) d \theta
$$

which is a general solution of equation (1) if $(y)_{0}=(\dot{y})_{0}=0$ (initial velocity of deflection and deflection $=0$ ). It must be remembered that $y$ is deflection, not displacement.

If we consider absolute motion (displacement) instead of relative motion (deflection), letting the ground displacement at any instant be $s(t),\left(j=\frac{d^{2} s}{d t^{2}}\right)$, we may rewrite equation (1):

$$
\frac{d^{2}}{d t^{2}}(y+s)+\lambda^{2}(y+s)=\lambda^{2} s
$$

where $(y+s)$ is the absolute displacement of the mass $m$. On comparing equation (4) with equation (1), we see immediately that $\frac{y+s}{\lambda^{2}}$ is the same function of $s$ that $y$ is of $-j$, or, for the boundary conditions:

$$
\begin{aligned}
& y=\dot{y}=s=\dot{s}=0 \text { at } t=0, \\
& \frac{y+s}{\lambda^{2}}=\frac{1}{\lambda} \int_{0}^{t} s(\theta) \sin \lambda(t-\theta) d \theta
\end{aligned}
$$

Consider next a simple oscillator with damping force proportional to $\dot{y}$ (rate of change of deflection), obeying the equation:

$$
\ddot{y}+2 \mu \dot{y}+\lambda^{2} y=-j
$$

where $\mu$ is proportional to the damping coefficient ( $2 m \mu$ equals the force produced by unit $\dot{y}$ ).

\footnotetext{
${ }^{2}$ This equation can be obtained in various ways; e.g., by the so-called "Variation of Parameter Method" of solving linear differential equations.
} 
For an oscillator with viscous damping dependent on rate of change of displacement $(\dot{y}+\dot{s})$, we obtain the following equation:

$$
\frac{d^{2}}{d t^{2}}(y+s)+2 \mu \frac{d}{d t}(y+s)+\lambda^{2}(y+s)=\lambda^{2} s
$$

It is apparent that the similarity between displacement and deflection equations previously found for undamped oscillators also holds in systems with viscous damping, providing the damping in the one case depends on $\dot{y}$, and in the other on $(\dot{y}+s)$.

We may solve equation (6) in exactly the same way as equation (1), obtaining:

where

$$
y(t)=-\frac{1}{\nu} \int_{0}^{t} j(\theta) e^{-\mu(t-\theta)} \sin \nu(t-\theta) d \theta
$$

$$
\nu^{2}=\lambda^{2}-\mu^{2}
$$

The solution of equation ( 7 ) will be similar.

Any analytical determination of the response of a simple linear oscillator to a given ground motion must be based on one of the preceding equations.

In carrying out this study experimentally the ordinary procedure is to construct a movable platform (shaking table), mounting on it an oscillator equivalent to that represented in figure (1). The table is then moved in accordance with the ground displacement to be studied, and the resulting motion of the oscillator recorded.

As previously stated, most of the usable records of severe ground motions during earthquakes are from accelerometers and are essentially records of ground acceleration. These records may be integrated twice to give displacements, but the process is very tedious. ${ }^{3}$ Furthermore, in this integration various discrepancies appear which must be arbitrarily eliminated. These, together with the errors unavoidable in such a calculation, make the over-all accuracy rather uncertain unless some kind of check is employed.

It has already been shown that $y$ and $\frac{y+s}{\lambda^{2}}$ are the same function of $(-j)$ and $(s)$, respectively. That is, for a given ground motion the corresponding oscillator deflection may be obtained experimentally in one of two ways: (1) by using a cam representing actual ground displacement, measuring the actual oscillator deflection; or (2), since $y$ is the same function of acceleration that $\frac{y+s}{\lambda^{2}}$ is of displacement, by using a cam of shape $j$ (proportional to recorded ground acceleration) to govern the shaking-table displacements, measuring the resulting oscillator displacement $(y+s)$, and dividing it by $\lambda^{2}$ to obtain the deflection $y$ which would result from an acceleration $j$.

\footnotetext{
${ }^{3}$ See, e.g., Chap. 3 (by Frank Neumann) of U. S. Dept. Commerce Special Publ. 201, "Earthquake Investigations in California, 1934-1935" (1936).
} 
If $F$ represents the restoring force in the oscillator, $F=k y=\lambda^{2} m y$. Therefore, in a linear oscillator the displacement of the mass due to an impressed base displacement $(j)$ will be numerically equal to that $F / m$ which would result from an acceleration of the same pattern $(j) . F / m$ may be considered as an equivalent static acceleration.

The only variation from present practice will be as follows. Instead of a cam representing ground displacement, the cam used must be shaped according to ground acceleration. If it is desired to consider viscous damping in the prototype dependent on deflection, the damping device in the model must act with respect to displacement instead. Finally, absolute displacements must be measured. These will be numerically equal to $F / m$ or to the equivalent static acceleration on the model.

Although this scheme, as just described, can only be used for simple linear oscillators, it will be seen that with some modifications it can also be used for oscillators with nonlinear friction. While there is yet no definite proof that the internal friction in an ordinary building cannot be closely approximated by viscous damping in a model, it is possible that such is the fact. That is, the energy-dissipating mechanism for the large deflections due to earthquakes may be somewhat different from that for the small deflections produced by a mechanical shaker within a building. If this is so, it is probable that for large deflections the friction can be assumed to depend on some power of velocity greater or less than unity. So-called solid or Coulomb friction results when this power is zero. On the other hand, damping forces proportional to the second power of velocity are not unusual. In general, the best approximation to actual conditions will be given by an exponent which is not an integer.

In any event, if the damping force in a particular case is known to be some function of the velocity of deflection, this case can be studied much as before (using a cam proportional to ground acceleration to actuate the shaking table), provided the damping force in the model is made to depend on absolute instead of relative velocity. Unfortunately, where nonviscous damping is concerned, the equations of motion are not linear. On this account there are certain limitations to be considered.

The general equations of motion corresponding to equations (6) and (7) are:

$$
\begin{gathered}
\ddot{y}+p(\dot{y})+\lambda^{2} y=-j \\
\frac{d^{2}}{d t^{2}}(y+s)+p(\dot{y}+\dot{s})+\lambda^{2}(y+s)=\lambda^{2} S
\end{gathered}
$$

where $p(\quad)$ signifies "function of."

Because of the nonlinearity of these equations it is necessary to revise the statement previously made regarding the correspondence of the relationship between $y$ and $(-j)$ and that between $(y+s)$ and $s$. This becomes: $y$ is the same function of $(-j)$ that $(y+s)$ is of $\lambda^{2} s$. This means that, if no changes are made in either time or length scales, the acceleration cam must be constructed with 
ordinates equal to $j / \lambda^{2}$. Thus for each oscillator frequency studied a separate cam must be used (or the same effect obtained by means of an adjustable coupling mechanism) if no other changes are made in the model. A possible alternative is to change the magnitude of damping without, of course, changing the law governing it. The justification of this is as follows. Since the use of a cam with an oscillator of a period for which the cam is not designed amounts to a distortion of the length scale, thus affecting unequally the different terms of equation (10), it is possible by adjusting the magnitude of the damping to restore balance to this equation. Then all terms of equation (10) will be increased or decreased in the same ratio, the ratio being $\frac{\lambda^{2}}{\lambda_{1}{ }^{2}}$, where $\frac{\lambda_{1}}{2 \pi}$ is the frequency for which the cam under consideration was constructed. Of course the measured deflections must then be divided by $\frac{\lambda^{2}}{\lambda_{1}{ }^{2}}$ to make them correspond with the original conditions.

An acceleration cam cannot be used in a model of a multistory building. For a system of several degrees of freedom, with different $\lambda$ 's, the fact that the displacement of each mode in one case is $\lambda^{2}$ the deflection in the other makes it practically impossible to calculate one response from the other. Here, as already mentioned, the responses of the various modes must be obtained separately, then combined.

Already mentioned is the difficulty of obtaining displacement records by double integration of accelerograms. Other advantages of the proposed scheme are the following. For the same amplitude of motion, smaller dimensions of model and smaller movements of the shaking table are necessary, because total rather than differential motions will be measured. Furthermore, absolute motion is somewhat simpler to record than is relative motion, since most of the recording mechanism can be at rest.

As a disadvantage of this scheme, it may be urged that an acceleration record is somewhat more complicated in shape and hence more difficult to use than a displacement record. The writers are not certain on this point, but believe that the objection will not be important. The reason for the smoothness of a displacement record is the fact that long-period waves are emphasized, tending to mask short-period waves which are reduced by integration. For an oscillator of given natural period, waves of approximately the same period are the most important. If these waves are masked by waves of much longer period, they cannot be adequately represented on a displacement cam. In any case, the allowable reduction in size of cam (since we must measure total rather than differential motion) will partly compensate for the relative complexity of an acceleration record.

California Institute of Techyology, Pasadena, Cattfornta. 